\title{
Spatial Assessment of Contact Between Humans and Anopheles and Aedes Mosquitoes in a Medium-Sized African Urban Setting, Using Salivary Antibody-Based Biomarkers
}

\author{
André B. Sagna, ${ }^{1,5,6}$ Daouda Kassié, ${ }^{2,7}$ Agnès Couvray, ${ }^{3}$ Akré Maurice Adja, ${ }^{5}$ Emmanuel Hermann, ${ }^{4}$ Gilles Riveau, ${ }^{4,6}$ Gérard Salem, ${ }^{3}$ Florence Fournet, ${ }^{1,7}$ and \\ Franck Remoue ${ }^{1}$ \\ ${ }^{1}$ MIVEGEC, University of Montpellier, CNRS, IRD, and ${ }^{2}$ ASTRE, University of Montpellier, CIRAD, INRA, Montpellier, ${ }^{3}$ CEPED, University of Paris Descartes, IRD, Paris, and ${ }^{4}$ CIIL, Institut Pasteur \\ de Lille, University of Lille, CNRS UMR, Inserm U1019, Lille, France; ${ }^{5}$ Institut Pierre Richet/Institut National de Santé Publique, Bouaké, Côte d'Ivoire; ${ }^{6}$ Biomedical Research Center Espoir Pour La \\ Santé, Saint-Louis, Sénégal; and ${ }^{7}$ Institut de Recherche en Sciences de la Santé, Bobo-Dioulasso, Burkina Faso
}

Background. Anarchic and poorly controlled urbanization led to an increased risk of mosquito-borne diseases (MBD) in many African cities. Here, we evaluate the spatial heterogeneity of human exposure to malaria and arboviral disease vectors in an urban area of northern Senegal, using antibody-based biomarkers of exposure to Anopheles and Aedes mosquito bites.

Methods. A cross-sectional study was undertaken during the rainy season of 2014 in 4 neighborhoods of Saint-Louis, a city in northern Senegal. Among children aged 6-59 months in each neighborhood, the dried blood spot technique was used to evaluate immunoglobulin G (IgG) responses to both gSG6-P1 (Anopheles) and Nterm-34-kDa (Aedes) salivary peptides as validated biomarkers of respective mosquito bite exposure.

Results. IgG response levels to gSG6-P1 and Nterm-34-kDa salivary peptides varied significantly between the 4 neighborhoods $(P<.0001)$. The level of exposure to Aedes bites also varied according to household access to sanitation services $(P=.027)$, whereas that of exposure to Anopheles bites varied according to insecticide-treated bed net use ( $P=.006)$. In addition, spatial clusters of high contact between humans and mosquitoes were identified inside 3 neighborhoods.

Conclusions. Antibody-based biomarkers of exposure to Anopheles and Aedes mosquito bites could be helpful tools for evaluating the heterogeneity of exposure to malaria and arboviral disease vectors by national control programs.

Keywords. Urban area; salivary biomarkers; gSG6-P1; Anopheles; Nterm-34-kD; Aedes; MBD risk

The overall prevalence of the major potentially life-threatening vector-borne diseases decreased by $6 \%$ between 2005 and 2015 [1]. The global prevalence of malaria and yellow fever, for example, decreased by $21 \%$ and $32 \%$, respectively, and the mortality rates attributed to these illnesses decreased, as well. However, vector-borne diseases remain one of the main causes of mortality and disability in most developing countries in tropical and subtropical areas.

While the available indicators are consistent with a decrease in the burden of vector-borne diseases, critical factors that could contribute to vector-borne diseases expanding into new habitats are increasingly being reported [2-4]. In subSaharan Africa, demographic characteristics are changing

Received 6 February 2019; editorial decision 28 May 2019; accepted 30 May 2019; published online June 1, 2019.

Correspondence: A. B. Sagna, Institut de Recherche pour le Développement, Montpellier, Hérault France (sagna.ab@gmail.com).

The Journal of Infectious Diseases ${ }^{\circledR} \quad$ 2019;XX:1-11

(C) The Author(s) 2019. Published by Oxford University Press for the Infectious Diseases Society of America. All rights reserved. For permissions, e-mail: journals.permissions@oup.com. DOI: 10.1093/infdis/jiz289 rapidly, with a growing number of people moving from rural to urban areas [5]. Rapid and anarchic urbanization combined with difficult socioeconomic conditions, such as inadequate housing, lack of public services, poor waste disposal, and poor water storage, can create ecological conditions that are conducive to mosquito breeding. Anopheles vectors of malaria, which until recently were confined to the bush and rural areas, are increasingly reported in African cities, owing to changes in the urban environment [6-8]. Urban malaria is becoming a serious concern, because most urban residents may lack immunity against malaria parasites and thus are at high risk of morbidity and mortality.

In Senegal, the malaria incidence decreased significantly during the past 10 years, from 1.5 million cases in 2006 to 174000 cases in 2009. Since 2009, the number of cases has remained low with annual variation, including 274119 cases in 2011, 265624 cases in 2014, and 395706 cases in 2017 [9, 10]. However, this decrease in malaria incidence is unequally distributed throughout the country. The northern regions show the lowest malaria incidence $(<5$ cases/1000), contrary to the southern regions [11]. Thus, the aim of the Senegalese National 
Malaria Control Program is to locally interrupt malaria transmission in the northern regions by 2020 [9]. However, malaria elimination efforts could be compromised if northern residents are always exposed to infected Anopheles bites. To go toward the elimination phase, it becomes essential to identify areas and individuals at risk for malaria transmission.

Concurrently to malaria, arboviruses such as dengue virus and chikungunya virus, transmitted by Aedes mosquitoes, are reemerging in southern countries, especially in urban cities. For instance, the global prevalence and mortality of dengue fever increased by $111 \%$ and 34\%, respectively, between 2005 and 2015 [12]. Global warming and the development of global transportation systems also seem to act as major factors that can influence vector-borne disease transmission $[2-4,13]$.

Several arboviruses, including dengue virus, chikungunya virus, yellow fever virus, Rift Valley fever virus, and West Nile virus, are circulating in Senegal [14-16], but their burden, clinical significance, and public health impact remain elusive, mostly because of the lack of diagnostic capacities for these viruses. An urban epidemic of dengue fever occurred during 2009 in Senegal, affecting 196 persons and including 5 cases of dengue hemorrhagic fever and 1 fatal case of dengue shock syndrome [17]. Another recent study reported widespread emergence of Rift Valley fever virus in Senegal during 2013-2014, affecting 11 persons, some of whom had severe infections with encephalitis and retinitis, and 52 animals [18]. This active and regular arboviral circulation emphasizes the need for moreeffective surveillance and control measures in this West African country.

To improve the surveillance and control of mosquito-borne diseases, people exposed to mosquito bites were assessed to develop immunological indicators of specific human antibody responses against salivary peptides from Aedes (Nterm-34-kDa) and Anopheles (gSG6-P1). These antibody-based biomarkers were validated by several studies as relevant tools to accurately evaluate (1) the risk of malaria [19-24] and arboviruses transmission [25, $26]$ in several settings, including urban areas, and (2) the effectiveness of different vector control strategies [27-30]. The aim of the present study was to evaluate the spatial heterogeneity of exposure to both vectors of malaria and arboviral diseases in a northern urban area of Senegal, using antibody-based biomarkers of human exposure to Anopheles and Aedes mosquito bites.

\section{METHODS}

\section{Ethics Statement}

This study followed ethical principles recommended by the Edinburgh revision of the Helsinki Declaration. The present study was performed after this approval by the National Ethics Committee of the Ministry of Health of Senegal (protocol SEN 14/29). Written informed consent from all parents or guardians of children who were enrolled in the study was obtained before inclusion.

\section{Study Location and Design}

The study was conducted in the urban city of Saint-Louis, located in the Senegal River Delta (northern Senegal). The climate is Sahelian (ie, tropical and dry) with an annual alternation between 2 contrasting seasons. A short rainy season with a relatively low rainfall amount (annual rainfall level, $<400 \mathrm{~mm}$ ) lasts from July to October, and a long dry season generally begins in November and ends in June.

A cross-sectional study was performed in August-September 2014 in 4 different neighborhoods of Saint-Louis: Pikine Sor Diagne (PSD), Léona (LEO), Guet Ndar (GND), and Ndioloffène (NDI). This study is part of a large comparative project, named SANTINELLES, in which 2 West African mid-sized cities, SaintLouis and Bobo-Dioulasso, Burkina Faso, were selected to examine the potential influence of diverse socioterritorial processes on population health. The 4 neighborhoods of Saint-Louis were selected according to the same geographic approach described by Kassié et al and used in Bobo-Dioulasso [31]. In brief, the topologic characteristics of Saint-Louis were determined by taking into account heterogeneity in the built environment and the distribution of urban infrastructure, such as healthcare structures and water fountains, based on interpretation of aerial photographs and satellite images. NDI and LEO are 2 old neighborhoods located in the central part of the town. Although they do not have a public health post, they benefit from those of surrounding neighborhoods [32]. Conversely, PSD is a recently established peripheral neighborhood, which remains under construction. Most of the neighborhood has no household access to water or sanitation services, unlike NDI and LEO. GND is an old peripheral neighborhood located on the island of Saint-Louis. Although the neighborhood has running water, wastewater and rainwater sewerage networks are absent.

Households to be surveyed were randomly selected using the sampling tool of ArcGis10 software and then localized. Information about the sanitary conditions of the household and household characteristics (house type and construction materials, bathroom type, access to running water, whether and how water was stored, access to sanitation services, and access to a sewage-disposal network) were recorded. In each included household, 1 child aged 5-59 months and 1 adult aged 35-59 years were randomly selected. Information about sex, age, and use of insecticide-treated mosquito nets (ITNs; ie, sleeping or not sleeping under an ITN) were collected, and selected individuals (around 40 individuals/day) were given an invitation card with their names and identifiers to join health centers for clinical surveys.

\section{Blood Sample Collection}

In collaboration with the academic inspection of Saint-Louis and the principals of schools, centers of examination were provisionally installed in a school in each of the 4 neighborhoods (Ministry of Education, authorization no. 000579/IEF/SLC,

2 - JID 2019:XX (XX XXXX) - Sagna et al 
obtained on 26 May 2014). Blood samples for the present serological analysis were collected on Whatman 3MM filter paper, using the dried blood spot (DBS) technique, and stored at $4^{\circ} \mathrm{C}$ until use.

\section{gSG6-P1 and Nterm-34-kDa Salivary Peptides}

The gSG6-P1 and Nterm-34-kDa salivary peptides were designed using bioinformatics as previously described [25, 33]. Both peptides were then synthesized and purified (purity, $>95 \%$ ) by Genepep (St-Jean de Vedas, France). Peptides were shipped in lyophilized form and then resuspended separately in milliQ water and stored in aliquots at $-20^{\circ} \mathrm{C}$ until their use.

\section{Enzyme-Linked Immunosorbent Assay (ELISA) Procedures}

Standardized DBSs (diameter, $1 \mathrm{~cm}$ ) were eluted as previously described [25]. ELISAs were performed on DBS eluates to separately assay immunoglobulin G (IgG) responses to both salivary antigens (gSG6-P1 and Nterm-34-kDa) as previously described $[25,34]$ but with some modifications (Supplementary Materials). The level of the anti-peptide IgG response was measured at the individual level and was expressed as the change in OD $(\Delta \mathrm{OD})$, calculated as ODx - ODn, where ODx and ODn represent the mean OD for 2 antigen wells and for 1 blank well containing no antigen, respectively.

\section{Statistical Analysis \\ Statistic Approach}

Statistical analyses were performed using Graph Pad Prism 5.0 (Graph Pad Software, San Diego, CA). The $\chi 2$ test was used to compare the rate of ITN use between neighborhoods. After checking the normality distribution using KolmogorovSmirnov and Shapiro Wilk tests, the nonparametric MannWhitney test was used to compare specific IgG antibody levels between 2 independent groups. Kruskal-Wallis test was used for the comparison of more than 2 groups. The Dunn post hoc test was used for multiple paired comparisons between neighborhoods. Significance was assigned at a $P$ value of $<.05$.

\section{Spatial Analysis}

All surveyed households were located using a global positioning system. From their geographical coordinates, a spatial database was created, and layers of information systems were then created. All of these layers were projected in WGS84 UTM 28N, the projection system used for Senegal. For spatial autocorrelation analysis, the hot spot analysis function (Getis-Ord $\mathrm{Gi}^{\star}$ ) of
ArcGIS, volume 10.0, was used to identify spatial aggregates in which surveyed individuals, by household, have a statistically significant level of exposure to mosquito bites (based on IgG levels) that was higher (denoting hot spots) or lower (denoting cold spots) than in a random distribution model. The statistical results were $z$ scores that were then mapped. Low $z$ scores were situated in cold spots, and high $z$ scores were in hot spots.

\section{RESULTS}

\section{Characteristics of the Study Population at the Household Scale}

Table 1 describes the characteristics of the study population during the study period. Overall, 1003 households were surveyed (234 in PSD, 241 in LEO, 272 in GND, and 256 in NDI), corresponding to 1648 individuals (834 children aged 6-59 months and 814 adults aged 32-59 years). For this specific serological study, only 809 children for whom a DBS was available were included. Participants from the 4 neighborhoods were comparable in median age and sex ratio. However, the rate of ITN use varied significantly according to neighborhood. GND possessed the lowest rate of ITN use, with only $11.8 \%$ of children (27 of 229) sleeping under an ITN, while it varied from 64.4\% (123 of 191) in PSD to 74.5\% (161 of 216) in NDI.

Household questionnaire analysis revealed that the great majority of households (82.6\% [668 of 809]) had connections installed for running water (Table 2). However, $98.9 \%$ of study households (800 of 809) stored water for daily activities, owing to frequent water cuts, and water in $97.3 \%$ (787) was mainly kept in covered containers. A total of $63.3 \%$ of households (512 of 809) did not have sewers for sewage disposal and, thus, discharged wastewater outdoors. However, this percentage varied significantly between the 4 neighborhoods $(P<.0001)$ : among households in PSD and GND, 89.0\% (170 of 191) and 93.5\% (216 of 231), respectively, discharged wastewater outdoors, compared with $42.7 \%$ (93 of 218) and 19.5\% (33 of 169 ) in NDI and LEO, respectively. In addition, GND was the only neighborhood where some households without toilets were found (11.3\% [26 of 231]; Table 2).

\section{Specific IgG Responses to the Nterm-34-kDa Salivary Peptide, According to Urban District}

Levels of IgG specific to Aedes Nterm-34-kDa salivary peptide were measured in children. Our results demonstrated that the level of specific IgG antibody varied significantly according to neighborhood $(P<.0001)$ and were higher in GND and NDI

Table 1. Descriptive Statistics of Participating Children in Saint-Louis, Senegal, by Neighborhood

\begin{tabular}{|c|c|c|c|c|c|}
\hline Characteristics & GND $(n=231)$ & $\mathrm{NDI}(\mathrm{n}=218)$ & $\operatorname{LEO}(n=169)$ & $\operatorname{PSD}(n=191)$ & $P$ \\
\hline Age, mo, median (range) & $27(6-60)$ & $34.5(5-60)$ & $31(6-60)$ & $29(5-59)$ & .057 \\
\hline Female sex, \% & 49.3 & 48.1 & 49.7 & 42.4 & .445 \\
\hline ITN use, \% (proportion) & $11.8(27 / 229)$ & $74.5(161 / 216)$ & $74.2(124 / 167)$ & $64.4(123 / 191)$ & $<.0001$ \\
\hline
\end{tabular}

Abbreviations: GND, Guet Ndar; ITN, insecticide-treated bed net; LEO, Léona; NDI, Ndioloffène; PSD, Pikine Sor Diagne. 
Table 2. Characteristics of Participating Households in Saint-Louis, Senegal, by Neighborhood

\begin{tabular}{|c|c|c|c|c|c|}
\hline Variable & GND $(n=231)$ & NDI $(n=218)$ & LEO $(n=169)$ & $\operatorname{PSD}(n=191)$ & Overall \\
\hline \multicolumn{6}{|l|}{ House construction material } \\
\hline Brick & $222(96.1)$ & $200(91.7)$ & $156(92.3)$ & 185 (96.9) & $763(94.3)$ \\
\hline Wooden & $4(1.7)$ & $7(3.2)$ & $0(0.0)$ & $3(1.6)$ & $14(1.7)$ \\
\hline Combined & $5(2.2)$ & $11(5.0)$ & $13(7.7)$ & $3(1.6)$ & $32(4.0)$ \\
\hline \multicolumn{6}{|l|}{ Bathroom facility } \\
\hline Flush toilet & 87 (37.7) & $119(54.6)$ & $89(52.7)$ & $102(53.4)$ & $397(49.1)$ \\
\hline Traditional latrine & $118(51.1)$ & $99(45.4)$ & $80(47.3)$ & $89(46.6)$ & $386(47.7)$ \\
\hline No facility & $26(11.3)$ & $0(0.0)$ & $0(0.0)$ & $0(0.0)$ & $26(3.2)$ \\
\hline \multicolumn{6}{|l|}{ Water supply } \\
\hline Tap & $208(90.0)$ & $213(97.7)$ & $164(97.0)$ & $83(43.5)$ & $668(82.6)$ \\
\hline Fountain & $19(8.2)$ & $2(0.9)$ & $0(0.0)$ & $102(53.4)$ & $123(15.2)$ \\
\hline Well & $0(0.0)$ & $2(0.9)$ & $0(0.0)$ & $4(2.1)$ & $6(0.7)$ \\
\hline Other & $4(1.7)$ & $1(0.5)$ & $5(3.0)$ & $2(1.0)$ & $12(1.5)$ \\
\hline \multicolumn{6}{|l|}{ Water storage container } \\
\hline Covered & $223(96.5)$ & $218(100)$ & $164(97.0)$ & 182 (95.3) & 787 (97.3) \\
\hline Uncovered & $0(0.0)$ & $0(0.0)$ & $1(0.6)$ & $0(0.0)$ & $1(0.1)$ \\
\hline Both covered and uncovered & $6(2.6)$ & $0(0.0)$ & $0(0.0)$ & $6(3.1)$ & $12(1.5)$ \\
\hline None & $2(0.9)$ & $0(0.0)$ & $4(2.4)$ & $3(1.6)$ & $9(1.1)$ \\
\hline \multicolumn{6}{|l|}{ Sewer } \\
\hline Public sewer & $3(1.3)$ & $55(25.2)$ & $127(75.1)$ & $7(3.7)$ & $192(23.7)$ \\
\hline Septic hole & $12(5.2)$ & $70(32.1)$ & $9(5.3)$ & $14(7.3)$ & $105(13.0)$ \\
\hline River/well/open space & $216(93.5)$ & $93(42.7)$ & $33(19.5)$ & $170(89.0)$ & $512(63.3)$ \\
\hline \multicolumn{6}{|l|}{ Garbage service } \\
\hline Municipal/EIG & $159(68.8)$ & $187(85.8)$ & 151 (89.3) & $84(44.0)$ & $581(71.8)$ \\
\hline Burned/open space/other & $72(31.2)$ & $31(14.2)$ & $18(10.7)$ & $107(56.0)$ & $228(28.2)$ \\
\hline
\end{tabular}

Data are no. (\%) of households, unless otherwise indicated.

Abbreviations: EIG, Economic Interest Group; GND, Guet Ndar; LEO, Léona; NDI, Ndioloffène; PSD, Pikine Sor Diagne.

as compared to PSD and LEO (Figure 1). The Dunn multiple comparison test indicated significant statistical differences in specific IgG levels between GND and LEO, GND and PSD, NDI and LEO, and NDI and PSD ( $P<.0001$ for all comparisons). In contrast, no significant differences in specific IgG levels between GND and NDI and between PSD and LEO were observed ( $P>.05$ for all comparisons).

Additionally, an association was found between access to sanitation services and IgG responses against the Aedes Nterm-34$\mathrm{kDa}$ peptide (Supplementary Figure 1). Indeed, the specific IgG level was significantly higher in neighborhoods where a higher percentage of households discharged wastewater outdoors (ie, GND and PSD), compared with neighborhoods where a lower percentage discharged wastewater outdoors (ie, LEO and NDI; $P=.027)$. No association was found between water storage or access to sanitation services and specific IgG levels.

The map of hot and cold spots based on IgG responses to Nterm-34-kDa indicated variations in the spatial distribution of IgG responses within neighborhoods. No cold spots were detected in GND and NDI (Figure 2). In NDI, the highest specific IgG levels (ie, hot spots of exposure) were located only in 4 households in the north (Ndioloffène Nord) and 1 household

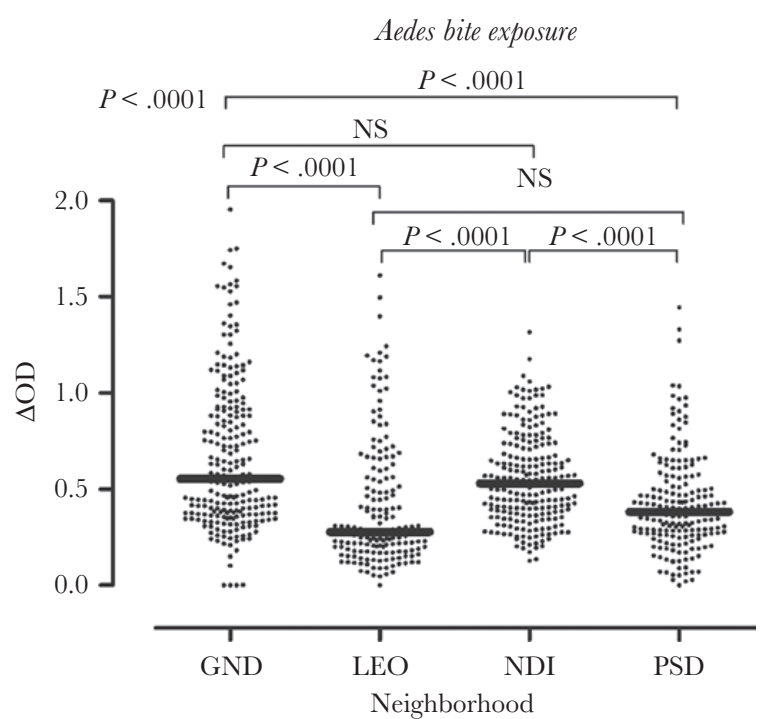

Figure 1. Levels of immunoglobulin $\mathrm{G}(\mathrm{lgG})$ specific to Nterm-34-kD salivary peptide in children in Saint-Louis, Senegal, by neighborhood. Black dots indicate individual lgG responses, and bars represent median values in each neighborhood. Statistically significant differences between the 4 neighborhoods $(P<.0001$, by the nonparametric Kruskal-Wallis test) and between all pairs of neighborhoods (by the Dunn post hoc test) are indicated. GND, Guet Ndar; LEO, Léona; NDI, Ndioloffène; NS, not significant; PSD, Pikine Sor Diagne; $\triangle O D$, change in $O D$. 


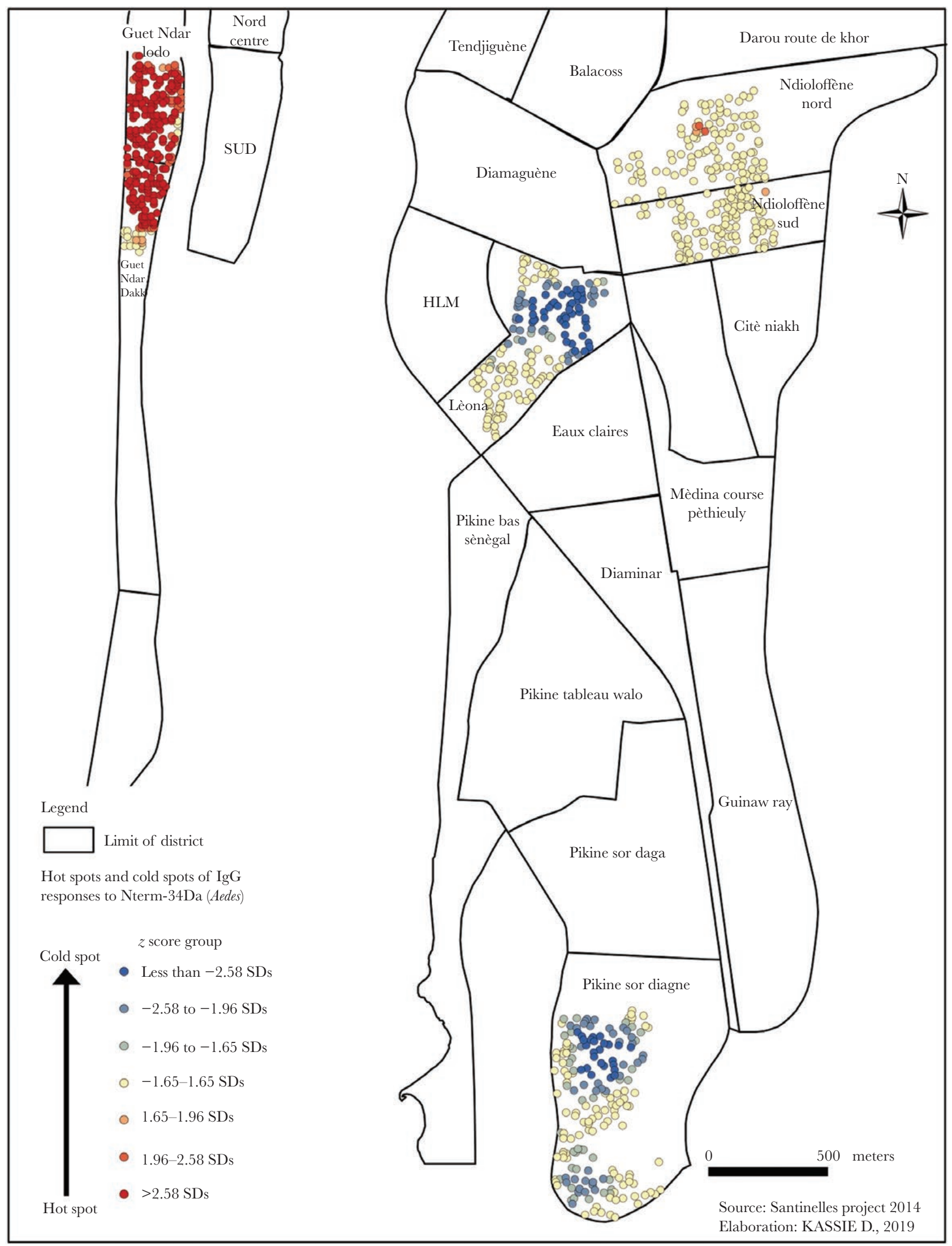

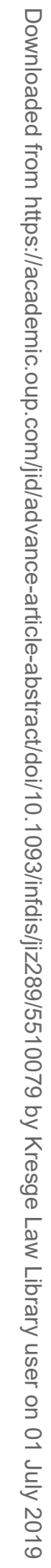

Figure 2. Hot and cold spots of human immunoglobulin G (lgG) responses to Aedes Nterm-34-kD salivary peptide in Saint-Louis, Senegal, by neighborhood. Each spot represents the IgG response to Nterm-34-kDa salivary peptide for 1 child in a participating household. 
in the south (Ndioloffène Sud). As for GND, children with a high IgG level were detected throughout the neighborhood. Conversely, LEO and PSD were characterized by low IgG levels (ie, cold spots of exposure). In LEO, cold spots were detected only in the central part of the neighborhood, whereas they were detected in the northern and southern parts of PSD.

\section{Specific IgG Responses to the gSG6-P1 Salivary Peptide, According to Urban District}

Anti-gSG6-P1 IgG levels were also assessed in the same children. As seen with the biomarker of exposure to Aedes, the levels of IgG responses against the Anopheles gSG6-P1 peptide varied significantly according to neighborhood $(P<.001)$ and were higher in GND, NDI, and PSD as compared to LEO (Figure 3). Of note, variations in IgG levels between neighborhoods seemed to be more marked for Aedes than for Anopheles.

In addition, an association was found between ITN use and IgG responses against the Anopheles gSG6-P1 peptide. The level of specific IgG responses was significantly higher in GND (where there was a low level of ITN use) as compared to NDI, LEO, and PSD combined ( $P=.006$; Supplementary Figure 2). No association was found between access to sanitation services and IgG levels to this Anopheles peptide.

The map of hot and cold spots based on IgG responses to gSG6-P1 also showed a spatial heterogeneity of specific IgG levels within neighborhoods (Figure 4). Hot spots of exposure to Anopheles bites were mainly located in the northern part of NDI and GND. No cold spots were detected in GND. In PSD, a

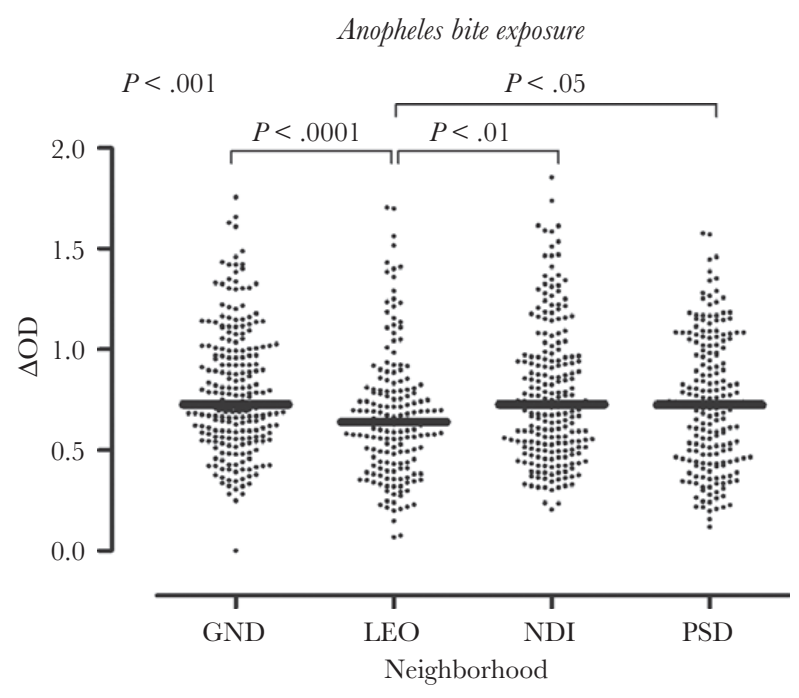

Figure 3. Immunoglobulin $\mathrm{G}(\mathrm{IgG})$ levels to Anopheles gSG6-P1 salivary peptide in children in Saint-Louis, Senegal, by neighborhood. Black dots indicate individual lgG responses, and bars represent median values in each neighborhood. Statistically significant differences between the four neighborhoods $(P<.001$, nonparametric Kruskal-Wallis test), between LEO and each of the other neighborhoods (Dunn's post-test) are indicated. GND, Guet Ndar; LEO, Léona; NDI, Ndioloffène; PSD, Pikine Sor Diagne. small group of residents with a high IgG level was located in the southern part of the neighborhood. Conversely, there were no hot spots of exposure to Anopheles in LEO, and cold spots were observed only in the central part of the neighborhood.

\section{DISCUSSION}

The present study evaluated the spatial heterogeneity of human exposure to Anopheles and Aedes vectors in a single city, using new, sensitive, specific, and individual antibodybased biomarkers. Although malaria and arboviral diseases are not transmitted by the same mosquito species, their geographic distribution in tropical areas overlaps, and vector control remains the main strategy for decreasing the burden of these MBDs. Simultaneous evaluation of exposure to Aedes and Anopheles could help identify individuals, groups of individuals, and/or areas with greater exposure to bites of these major vectors and, thus, where the risks of malaria and arbovirus transmission are greatest. This would lead to the development of an integrated vector management program that takes into account the spatial heterogeneity of the risk of MBD transmission, to optimize the program's efficacy and make better use of available funds for targeting multiple MDB by means of 1 or more interventions [35].

Our study shows that children aged $<5$ years were exposed to Aedes bites and that the level of exposure significantly varied between the 4 neighborhoods. In addition, areas of high contact between children and Aedes mosquitoes were located only in a small patch in the northern part of NDI, whereas they were dispersed throughout GND. This suggests that human-vector contacts might preferentially occur in peridomestic and/or domestic environments, because children aged $<5$ years are less mobile, and could be linked to different human behaviors, socioeconomic factors, levels of urbanization [36], presence of vegetation, and/or breeding sites in and around households between neighborhoods. A recent study in the Americas showed that antibody responses to Aedes aegypti saliva were significantly higher in people living in households positive for Aedes mosquito larvae [37]. The clustered hot spot of Aedes bite exposures in NDI, which is relatively well endowed with materials characteristic of urban environments, could then be explained by a concentrated number of breeding sites (eg, cans, buckets, tires, and wells) in and around households discharging wastewater outdoors. On the contrary, GND is devoid of a sewage-disposal network, with nearly all inhabitants discharging wastewater outdoors. In addition, GND has the highest population density in Saint-Louis, with $>16000$ inhabitants in a 16.9-hectare area [38], and the highest building density. All of these factors have previously been shown to be associated with a high A. aegypti density in urban areas [39] and could likely increase humanvector contact throughout the neighborhood.

Surprisingly, children from NDI were as highly exposed to Aedes bites as those from GND, despite obvious differences

6 - JID 2019:XX (XX XXXX) - Sagna et al 


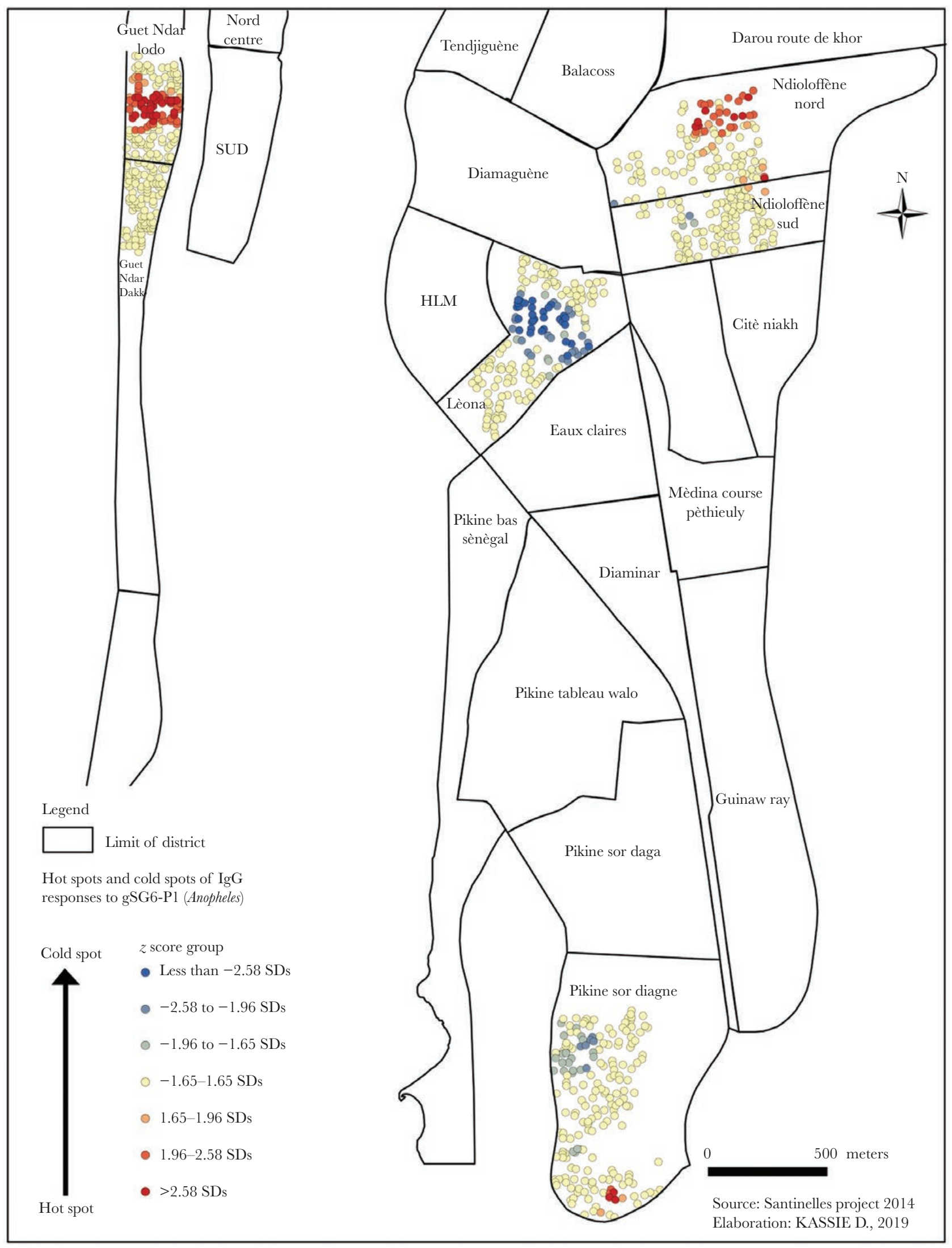

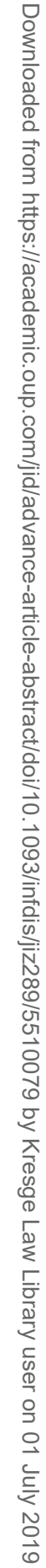

Figure 4. Hot and cold spots of human immunoglobulin G (IgG) responses to Anopheles gSG6-P1 salivary peptide in Saint-Louis, Senegal, by neighborhood. Each spot represents the IgG response to gSG6-P1 salivary peptide for 1 child in a participating household. 
between the characteristics of these 2 neighborhoods. The reason for the high Aedes exposure in children from NDI is unknown. Further studies on human and environmental factors affecting the $A$. aegypti distribution are needed to better understand the high level of Aedes exposure observed in NDI.

Although the prevalence proportions for most arboviral diseases are higher for individuals aged 5-15 years, this study indicates that children aged $0-5$ years were quite highly exposed to Aedes mosquitoes. A high level of human-Aedes contact has been shown to be associated with the risk of arboviral transmission [40]. In addition, a study in Thailand reported an increasing flavivirus seroprevalence from ages 1-2 years to ages 6-9 years [41], suggesting that flavivirus infection occurs at an early age. The evaluation of Aedes contacts among young children could provide information on the level of exposure to Aedes bites in each neighborhood, as older children and adults may be exposed to Aedes elsewhere than in their neighborhoods. These data showed that children aged $<5$ years are not safe from Aedes mosquito bites and that arboviral disease control efforts should also target young children.

Regarding the exposure to Anopheles bites, our results indicated that GND, NDI, and PSD were more exposed to malaria vectors than LEO. This high level of exposure to Anopheles in NDI and PSD was surprising, given the high rate of ITN use in both neighborhoods. Indeed, some studies have shown that adequate use of ITNs leads to a decreased antibody response to gSG6-P1 salivary peptide [34]. However, factors such as the improper use of or presence of holes in ITNs could also explain the high exposure to mosquito vector bites in children from NDI and PSD despite the high rate of ITN use. Indeed, a study in Kenya detected Anopheles gambiae sensu lato in ITNs without holes [42]. Noukpo et al [29] also showed that children sleeping under ITNs with holes had a higher antibody response to the gSG6-P1 peptide than those sleeping under ITNs without holes. However, the authors did not control for the effective use of ITNs by individuals in households and for the physical integrity of ITNs (ie, the presence of holes).

Furthermore, hot spots of Anopheles bite exposure inside neighborhoods were located in the northern part of GND and NDI and in the southern part of PSD. The presence of these hot spots could be explained by several individual and/or household factors, distance from breeding sites [43], and vegetation cover [44]. For instance, the particularly low dispersion of adult mosquitoes in urban areas could lead to high exposure among children living near Anopheles breeding sites, compared with exposure among those who live further away [36, 45, 46]. The occurrence of malaria in African cities has also been linked to agricultural practices [5,47]. Thus, the presence of vegetable gardens in NDI, for example, could be a likely explanation of the level of exposure to Anopheles bites among children [48]. These results indicate that people with high levels of exposure to Anopheles could be extremely focused in some areas inside neighborhoods. To eliminate malaria in this part of the country, it becomes critical for the Senegalese National Malaria Control Program to accurately locate these hot spots of high humanAnopheles contact to better orient and adequately implement vector control strategies.

Our results also demonstrate that children living in the northern part of GND and NDI were highly exposed to both Aedes and Anopheles bites and, thus, exposed to the risk of both malaria and arbovirus transmission. Concurrent infections with malaria parasites and arboviruses were previously reported in patients infected with arboviruses in southeastern Senegal [15, 49]. Also, it has been shown that dengue virus and malaria parasite coinfection is more severe than monoinfection with either pathogen [50]. Control programs should therefore combined their efforts, to effectively fight both mosquito vectors.

In conclusion, our study indicated that a high level of human-mosquito contact occurred in Saint-Louis. This humanmosquito contact was heterogeneous and extremely focused in some parts of the neighborhoods. In addition, areas with concurrent high exposure to both Anopheles and Aedes mosquitoes were identified in the neighborhoods of GND and NDI. These findings call for surveillance of both Anopheles and Aedes mosquitoes and the development of integrated vector-control strategies that can simultaneously control vector-borne disease due to both mosquito species. The use of salivary biomarkers of human exposure could help control programs identify individuals, groups, or districts who are highly exposed to malaria and arboviral disease vectors and, thus, at risk for diseases due to these pathogens.

\section{SUPPLEMENTARY DATA}

Supplementary materials are available at The Journal of Infectious Diseases online. Consisting of data provided by the authors to benefit the reader, the posted materials are not copyedited and are the sole responsibility of the authors, so questions or comments should be addressed to the corresponding author.

\section{Notes}

Acknowledgments. We thank the populations of the 4 participating neighborhoods of Saint-Louis, especially householders and guardians of children, for their kind support and great collaboration; Mr Simon Senghor, Ms Lydie Bandagny, and Ms Daba Diagne, for their technical support during field study and blood sample collection; Dr Mawlouth Diallo and Dr Ibrahima Dia, for their technical support during entomological data collection.

F. R., F. F., G. S., G. R., and E. H. conceived the study. A. B. S., D. K., and A. C. collected data in field surveys. A. B. S. performed all ELISAs and drafted the first manuscript. D. K. performed spatial mapping and the GIS study. A. B. S. and D. K. worked on the statistical analysis. F. R. and A. M. A. participated in

8 - JID 2019:XX (XX XXXX) - Sagna et al 
preparation and writing of the manuscript and revised the manuscript. F. R. and F. F. provided guidance on revision of the manuscript. All authors read and approved the final manuscript.

Financial support. This work was supported by the Agence Nationale de la Recherche (ANR12-INEG-007) and the Méditerannée Infection Foundation (postdoctoral fellowship to A. B. S.).

Potential conflicts of interest. All authors: No reported conflicts.

All authors have submitted the ICMJE Form for Disclosure of Potential Conflicts of Interest. Conflicts that the editors consider relevant to the content of the manuscript have been disclosed.

\section{References}

1. GBD 2015 Disease and Injury Incidence and Prevalence Collaborators. Global, regional, and national incidence, prevalence, and years lived with disability for 310 diseases and injuries, 1990-2015: a systematic analysis for the Global burden of disease study 2015. Lancet 2016; 388:1545-1602.

2. Liang G, Gao X, Gould EA. Factors responsible for the emergence of arboviruses; strategies, challenges and limitations for their control. Emerg Microbes Infect 2015; 4:e18.

3. Hassell JM, Begon M, Ward MJ, Fèvre EM. Urbanization and disease emergence: dynamics at the wildlife-livestockhuman interface. Trends Ecol Evol 2017; 32:55-67.

4. Gould E, Pettersson J, Higgs S, Charrel R, de Lamballerie X. Emerging arboviruses: why today? One Health 2017; 4:1-13.

5. Donnelly MJ, McCall PJ, Lengeler C, et al. Malaria and urbanization in sub-Saharan Africa. Malar J 2005; 4:12.

6. Byrne N. Urban malaria risk in sub-Saharan Africa: where is the evidence? Travel Med Infect Dis 2007; 5:135-7.

7. Matthys B, N'Goran EK, Koné M, et al. Urban agricultural land use and characterization of mosquito larval habitats in a medium-sized town of Côte d'Ivoire. J Vector Ecol 2006; 31:319-33.

8. Mourou JR, Coffinet T, Jarjaval F, et al. Malaria transmission in Libreville: results of a one year survey. Malar J 2012; $11: 40$.

9. PNLP. Plan Stratégique National 2016-2020. Sénégal: Programme National de Lutte contre le Paludisme, 2016:171. http://www.pnlp.sn/wp-content/ uploads/2016/08/PNLP_PSN_VFF_03-02-2016.pdf. Accessed on September 01, 2018.

10. PNLP. Enquête nationale sur les indicateurs du paludisme au Sénégal. Sénégal: Programme National de Lutte contre le Paludisme; 2017:191. http://www. pnlp.sn/wp-content/uploads/2018/01/Rapport-Final_ ENP_2016-vs_10_04_2017.pdf. Accessed on September $01,2018$.
11. Thwing J, Eckert E, Dione DA, et al. Declines in malaria burden and all-cause child mortality following increases in control interventions in Senegal, 2005-2010. Am J Trop Med Hyg 2017; 97(Suppl 3):89-98.

12. GBD 2015 Mortality and Causes of Death Collaborators. Global, regional, and national life expectancy, all-cause mortality, and cause-specific mortality for 249 causes of death, 1980-2015: a systematic analysis for the Global Burden of Disease Study 2015. Lancet 2016; 388:1459-1544.

13. Tjaden NB, Suk JE, Fischer D, Thomas SM, Beierkuhnlein C, Semenza JC. Modelling the effects of global climate change on Chikungunya transmission in the $21^{\text {st }}$ century. Sci Rep 2017; 7:3813.

14. Chevalier V, Lancelot R, Diaité A, Mondet B, Sall B, De Lamballerie X. Serological assessment of West Nile fever virus activity in the pastoral system of Ferlo, Senegal. Ann N Y Acad Sci 2006; 1081:216-25.

15. Sow A, Loucoubar C, Diallo D, et al. Concurrent malaria and arbovirus infections in Kedougou, southeastern Senegal. Malar J 2016; 15:47.

16. Amarasinghe A, Kuritsk JN, Letson GW, Margolis HS. Dengue virus infection in Africa. Emerg Infect Dis 2011; 17:1349-54.

17. Faye $\mathrm{O}, \mathrm{Ba} \mathrm{Y}$, Faye $\mathrm{O}$, et al. Urban epidemic of dengue virus serotype 3 infection, Senegal, 2009. Emerg Infect Dis 2014; 20:456-9.

18. Sow A, Faye O, Ba Y, et al. Widespread Rift Valley fever emergence in Senegal in 2013-2014. Open Forum Infect Dis 2016; 3: ofw149.

19. Sagna AB, Sarr JB, Gaayeb L, et al. gSG6-P1 salivary biomarker discriminates micro-geographical heterogeneity of human exposure to Anopheles bites in low and seasonal malaria areas. Parasit Vectors 2013; 6:68.

20. Sagna AB, Gaayeb L, Sarr JB, et al. Plasmodium falciparum infection during dry season: IgG responses to Anopheles gambiae salivary gSG6-P1 peptide as sensitive biomarker for malaria risk in Northern Senegal. Malar J 2013; 12:301.

21. Drame PM, Machault V, Diallo A, et al. IgG responses to the gSG6-P1 salivary peptide for evaluating human exposure to Anopheles bites in urban areas of Dakar region, Sénégal. Malar J 2012; 11:72.

22. Poinsignon A, Cornelie S, Ba F, et al. Human IgG response to a salivary peptide, gSG6-P1, as a new immunoepidemiological tool for evaluating low-level exposure to Anopheles bites. Malar J 2009; 8:198.

23. Badu K, Siangla J, Larbi J, et al. Variation in exposure to Anopheles gambiae salivary gland peptide (gSG6-P1) across different malaria transmission settings in the western Kenya highlands. Malar J 2012; 11:318.

24. Londono-Renteria B, Drame PM, Weitzel T, et al. An. gambiae gSG6-P1 evaluation as a proxy for human-vector 
contact in the Americas: a pilot study. Parasit Vectors 2015; $8: 533$.

25. Elanga Ndille E, Doucoure S, Damien G, et al. First attempt to validate human IgG antibody response to Nterm-34kDa salivary peptide as biomarker for evaluating exposure to Aedes aegypti bites. PLoS Negl Trop Dis 2012; 6:e1905.

26. Ndille EE, Dubot-Pérès A, Doucoure S, et al. Human IgG antibody response to Aedes aegypti Nterm-34 kDa salivary peptide as an indicator to identify areas at high risk for dengue transmission: a retrospective study in urban settings of Vientiane city, Lao PDR. Trop Med Int Health 2014; 19:576-80.

27. Drame PM, Diallo A, Poinsignon A, et al. Evaluation of the effectiveness of malaria vector control measures in urban settings of Dakar by a specific anopheles salivary biomarker. PLoS One 2013; 8:e66354.

28. Corbel V, Akogbeto M, Damien GB, et al. Combination of malaria vector control interventions in pyrethroid resistance area in Benin: a cluster randomised controlled trial. Lancet Infect Dis 2012; 12:617-26.

29. Noukpo MH, Damien GB, Elanga-N'Dille E, et al. Operational assessment of long-lasting insecticidal nets by using an Anopheles salivary biomarker of human-vector contact. Am J Trop Med Hyg 2016; 95:1376-82.

30. Elanga Ndille E, Doucoure S, Poinsignon A, et al. Human IgG antibody response to Aedes Nterm-34 kDa salivary peptide, an epidemiological tool to assess vector control in Chikungunya and Dengue transmission area. PLoS Negl Trop Dis 2016; 10:e005109.

31. Kassié D, Roudot A, Dessay N, Piermay JL, Salem G, Fournet F. Development of a spatial sampling protocol using GIS to measure health disparities in Bobo-Dioulasso, Burkina Faso, a medium-sized African city. Int J Health Geogr 2017; 16:14.

32. Vialard L, Squiban C, Riveau G, et al. Correction: Vialard, L., et al. toward a socio-territorial approach to health: health equity in west Africa. Int J Environ Res Public Health 2017; 14:106. .

33. Poinsignon A, Cornelie S, Mestres-Simon M, et al. Novel peptide marker corresponding to salivary protein gSG6 potentially identifies exposure to Anopheles bites. PLoS One 2008; 3:e2472.

34. Drame PM, Poinsignon A, Besnard P, et al. Human antibody responses to the Anopheles salivary gSG6-P1 peptide: a novel tool for evaluating the efficacy of ITNs in malaria vector control. PLoS One 2010; 5:e15596.

35. Beier JC, Keating J, Githure JI, Macdonald MB, Impoinvil DE, Novak RJ. Integrated vector management for malaria control. Malar J 2008; 7(Suppl 1):S4.

36. Baragatti M, Fournet F, Henry MC, et al. Social and environmental malaria risk factors in urban areas of Ouagadougou, Burkina Faso. Malar J 2009; 8:13.
37. Londoño-Rentería B, Cárdenas JC, Giovanni JE, et al. Aedes aegypti anti-salivary gland antibody concentration and dengue virus exposure history in healthy individuals living in an endemic area in Colombia. Biomedica 2015; 35:572-81.

38. DUA. Plan Directeur d'Urbanisme (PDU) - Saint-Louis Horizon 2025 [Internet]. Sénégal: Direction de l'Urbanisme et de l'Architecture, 2008:93. http://www.pdudakar.gouv.sn/ PDU-de-Saint-Louis.html. Accessed on January 17, 2019.

39. Cox J, Grillet ME, Ramos OM, Amador M, Barrera R. Habitat segregation of dengue vectors along an urban environmental gradient. Am J Trop Med Hyg 2007; 76:820-6.

40. Ndille EE, Dubot-Pérès A, Doucoure S, et al. Human IgG antibody response to Aedes aegypti Nterm-34 kDa salivary peptide as an indicator to identify areas at high risk for dengue transmission: a retrospective study in urban settings of Vientiane city, Lao PDR. Trop Med Int Health 2014; 19:576-80.

41. Bartley LM, Carabin H, Vinh Chau N, et al. Assessment of the factors associated with flavivirus seroprevalence in a population in Southern Vietnam. Epidemiol Infect 2002; 128:213-20.

42. Ochomo EO, Bayoh NM, Walker ED, et al. The efficacy of long-lasting nets with declining physical integrity may be compromised in areas with high levels of pyrethroid resistance. Malar J 2013; 12:368.

43. Stoler J, Weeks JR, Getis A, Hill AG. Distance threshold for the effect of urban agriculture on elevated self-reported malaria prevalence in Accra, Ghana. Am J Trop Med Hyg 2009; 80:547-54.

44. Traoré DF, Sagna AB, Adja AM, et al. Exploring the heterogeneity of human exposure to malaria vectors in an urban setting, Bouaké, Côte d'Ivoire, using an immunoepidemiological biomarker. Malar J 2019; 18:68.

45. Machault V, Gadiaga L, Vignolles C, et al. Highly focused anopheline breeding sites and malaria transmission in Dakar. Malar J 2009; 8:138.

46. Van Der Hoek W, Konradsen F, Amerasinghe PH, Perera D, Piyaratne MK, Amerasinghe FP. Towards a risk map of malaria for Sri Lanka: the importance of house location relative to vector breeding sites. Int J Epidemiol 2003; 32:280-5.

47. Klinkenberg E, McCall PJ, Hastings IM, Wilson MD, Amerasinghe FP, Donnelly MJ. Malaria and irrigated crops, Accra, Ghana. Emerg Infect Dis 2005; 11:1290-3.

48. Robert V, Awono-Ambene HP, Thioulouse J. Ecology of larval mosquitoes, with special reference to Anopheles arabiensis (Diptera:Culcidae) in market-garden wells in urban Dakar, Senegal. J Med Entomol 1998; 35:948-55.

49. Niang M, Loucoubar C, Sow A, et al. Genetic diversity of Plasmodium falciparum isolates from concurrent malaria and arbovirus co-infections in Kedougou, southeastern Senegal. Malar J 2016; 15:155.

50. Epelboin L, Hanf M, Dussart P, et al. Is dengue and malaria co-infection more severe than single infections? A retrospective matched-pair study in French Guiana. Malar J 2012; 11:142.

10 • JID 2019:XX (XX XXXX) • Sagna et al 